\title{
MicroRNA-30a downregulation contributes to chemoresistance of osteosarcoma cells through activating Beclin-1-mediated autophagy
}

\author{
RUIDA XU, SHUZHONG LIU, HAIHONG CHEN and LIFENG LAO \\ Department of Orthopaedic Surgery, Renji Hospital, Shanghai Jiaotong University \\ School of Medicine, Shanghai 200127, P.R. China
}

Received October 20, 2015; Accepted November 26, 2015

DOI: $10.3892 /$ or.2015.4497

\begin{abstract}
Autophagy has been recognized as an important element of tumor cell migration, invasion, and chemoresistance, and our previous results showed that Beclin-1-mediated autophagy contributed to osteosarcoma chemoresistance. However, the regulating mechanism of autophagy is still unclear. In this study, our aim was to clarify microRNA (miRNA)-related mechanisms underlying Beclin-1-mediated autophagy followed by chemotherapy in osteosarcoma. First, miRNA screening using qRT-PCR identified that miR-30a was significantly reduced in Dox-resistant osteosarcoma cells. Second, the autophagy activity in Dox-resistant increased while miR-30a expression reduced after chemotherapy agents as indicated by the enhanced expression of Beclin-1, the increased conversion of microtubule-associated protein LC3-I to LC3-II. Furthermore, overexpression of miR-30a significantly promoted chemotherapy-induced apoptosis and reduced autophagy activity responding to chemotherapy. Moreover, rapamycin, an autophagy promoter was able to partly reverse the effect of miR-30a and Luciferase reporter assay identified that miR-30a directly binds to the 3'-UTR of Beclin-1 gene, which further confirmed that miR-30a reduced chemoresistance via suppressing Beclin-1-mediated autophagy. Collectively these results indicate miR-30a and its downstream target gene Beclin-1 can be used in treatment of osteosarcoma chemoresistance in the future.
\end{abstract}

\section{Introduction}

Osteosarcoma is one of the most common malignant bone tumor in childhood (1), but it is difficult to cure, especially

Correspondence to: Professor Lifeng Lao, Department of Orthopaedic Surgery, Renji Hospital, Shanghai Jiaotong University School of Medicine, Shanghai 200127, P.R. China

E-mail: renjispine@163.com

Key words: microRNA-30a, autophagy, Beclin-1, chemoresistance during the later stages. Chemoresistance is a main cause of unsatisfactory survival outcomes, although chemotherapy has become more effective in recent years $(2,3)$. Several factors are implicated in the development of chemoresistance including genetic alterations, drug uptake and increased activation of DNA repair mechanisms and evasion of chemotherapyinduced apoptosis $(4,5)$. Additionally, our previous results showed that Beclin-1-mediated autophagy contributed to osteosarcoma chemoresistance (6). However, the molecular regulating mechanism of autophagy in chemoresistance is largely unclear.

MicroRNA (miRNA), an endogenous noncoding RNA with 18-25 nucleotides, is able to regulate gene expression by binding to the 3'-untranslated region (3'-UTR) of their target mRNAs, modulating mRNA and protein expression. A series studies on miRNAs in osteosarcoma have been performed and several miRNAs contribute to the development and progression of osteosarcoma (2,7-9). miR-30a, an intronic transcriptional unit, was widely expressed in various tissues (10). Recent studies have suggested that miR-30a played a tumorsuppressive role in various malignant tumors, including lung cancer (11), breast cancer (12), colon cancer (13), and nasopharyngeal carcinoma (14). Moreover, a negative correlation was observed between miR-30a expression and malignant grade in osteosarcoma. Importantly, overexpression of miR-30a reduced proliferation, migration, and invasion and further studies revealed that runt-related transcription factor 2 was a regulative target gene of miR-30a (7). However, the role of miR-30a in osteosarcoma chemoresistance remains elusive.

Autophagy is essential for the maintenance of cellular biosynthesis, growth and differentiation. Accumulating evidence supports that activation of autophagy was also a crucial step in the process of chemoresistance (15). Since autophagy promoted tumor cells to survive under adverse stress conditions, such as hypoxia, ischemia and chemotherapy (16). Recent studies indicated microRNA-143 (17), miR-101 (18), and miR-22 (19) to be involved in chemoresistance through regulating autophagy. Therefore, a better understanding of the role of miRNA-mediated autophagy in osteosarcoma is essential for future treatment in chemoresistance.

In this study, we investigated the expression of miR-30a in osteosarcoma cell lines. We demonstrated autophagy 
was activated by chemotherapy and miR-30a was downregulated following chemotherapy. Further study showed that overexpression of miR-30a reduced chemoresistance of osteosarcoma cells through downregulating autophagy. In addition, Beclin-1 was identified as the target gene of miR-30a in osteosarcoma. The above results indicate miR-30a and its downstream target gene Beclin-1 can be used in treatment of osteosarcoma chemoresistance in the future.

\section{Materials and methods}

Cell culture and doxorubicin-resistant MG-63 cell line. Human osteosarcoma MG-63 cell line was preserved in our laboratory as previously described (20). Cells was cultured in high glucose DMEM medium supplemented with $10 \%$ fetal bovine serum (Gibco, Carlsbad, CA, USA), $100 \mathrm{U} / \mathrm{ml}$ penicillin, and $100 \mu \mathrm{g} / \mathrm{ml}$ streptomycin, and were cultured in a humidified atmosphere containing $5 \% \mathrm{CO}_{2}$ at $37^{\circ} \mathrm{C}$. A doxorubicin (Dox)-resistant cell line (MG-63/Dox) was established from its parental cell line MG-63 by gradually increasing the concentration of Dox over 6 months. The concentration of Dox added to MG-63 cells was from 10 to $100 \mathrm{nM}$, after which the cells were maintained in culture medium containing $100 \mathrm{nM}$ Dox, it displayed 6-fold resistance to Dox compared with the corresponding parental sensitive cells.

Quantitative real-time RT-PCR. Total RNA containing miRNA was extracted from cells with TRIzol Reagent (Invitrogen, Carlsbad, CA, USA) according to the manufacturer's instructions. miR-30a and U6 small nuclear 2 (U6) expression was detected from the cDNA product using TaqMan miRNA sequence-specific probes (Takara, Japan) and ABI Prism 7500 sequence detection system (Applied Biosystems, Foster City, CA, USA). The fold-change in gene expression was calculated by $2^{-\Delta \Delta \mathrm{CT}}$.

Overexpression of miR-30a. To generate the stable cell line, MG-63/Dox osteosarcoma cells were transfected with lentiviruses containing miR-30a gene (Lenti-miR-30a) or a blank lentivirus expression vectors (Lenti-NC) GeneChem Corp. (Shanghai, China). The infection efficiency was confirmed by qRT-PCR after transfection $72 \mathrm{~h}$.

miRNA target prediction. Candidate targets of miR-30a and Beclin-1 were predicted by miRBase (http://www.mirbase. org/) and TargetScan (http://www.Targetscan.org/).

Cell proliferation and apoptosis assays. Cell proliferation was evaluated using Cell Counting Kit-8 (CCK-8, Beyotime Institute of Biotechnology, Haimen, China). Cells $\left(2 \times 10^{3}\right)$ were seeded in 96-well plates and cultured for 1, 2, 3, 4 and 5 days after transfection. CCK-8 solution $(10 \mu \mathrm{l})$ was added to each well and followed by incubation for $2 \mathrm{~h}$. Absorbance was measured at $450 \mathrm{~nm}$ with a Microplate Autoreader (Bio-Rad, Hercules, CA, USA). For apoptosis detection, miR-30a transfected cells in the presence or absence of chemotherapy agents were stained with Annexin V/PI double staining kit (BD Biosciences, Bedford, MA, USA) according to the manufacturer's protocol. Apoptotic cells were examined by flow cytometry (6).
Western blot analysis. Proteins were separated by $10 \%$ SDS pages and then performed as previously described (6). Antibodies against Beclin-1, p62, LC3-I, LC3-II, cleaved caspase-3, Total caspase-3, and $\beta$-actin were purchased from Cell Signaling Technology, Inc. (Danvers, MA, USA). Then the band were incubated by horseradish peroxidase-labeled goat anti-rabbit IgG (Santa Cruz Biotechnology, Santa Cruz, CA, USA) and detected by chemiluminescence. $\beta$-actin was used as a protein loading control.

Stably expressing GFP-LC3. The adenovirus vector containing the GFP-LC3 reporter was purchased from Hanbio (Shanghai, China). In the presence or absence of chemotherapy, the cells were analyzed by a fluorescence microscope (Olympus, Tokyo, Japan).

Luciferase reporter assay. According to the expression of miR-30a with the Beclin-1 3'-UTR, 3'-UTR sequence or three copies of the mutated 3'-UTR sequence of Beclin-1 were amplified by PCR from human genomic DNA and immediately inserted into pGL3-control vector (Promega Corp., WI, USA). MG-63/Dox cells in 6-well plates were transfected with $1 \mu \mathrm{g}$ of the firefly luciferase report vector, $1 \mathrm{~h}$ post-transfection with $25 \mathrm{nM}$ of mimic NC, miR-30a mimics, anti-NC or anti-miR-30a. At $24 \mathrm{~h}$ post-transfection, firefly luciferase activities were measured consecutively using luciferase assays (Promega Corp.).

Statistical analysis. The data are expressed as means \pm standard error (SE). A two-tailed Student's t-test was used to determine the significance of differences between groups. Results with P-values $<0.05$ were considered statistically significant and SPSS 13.0 was used for the statistical analysis.

\section{Results}

Autophagy is increased in Dox-resistant osteosarcoma cells and miR-30a is downregulated. Dox has been widely used in clinical chemotherapy of osteosarcoma patients, we established the Dox-resistant MG-63 cells. In terms of $\mathrm{IC}_{50}$, the Dox-selected MG-63/Dox subline showed 6-fold higher resistance to doxorubicin (Dox), 2.2-fold, and 2-fold higher resistance to cisplatin (Cis), and methotrexate (Mtx), respectively, when compared with parental MG-63cells. The results indicated that MG-63/Dox subline was cross-resistant to the conventional chemotherapeutic agents (Fig. 1A).

Next, we assessed the autophagy activity of the above two different osteosarcoma cells, by using LC3-GFPlabeled autophagosomes, which indicated that the level of autophagy was significantly increased in MG-63/Dox subline cells (Fig. 1B). Western blot analysis confirmed that the autophagy-related protein Beclin-1 increased in MG-63/Dox cells compared with the control cells (Fig. 1C). The autophagy-related protein LC3-I and LC3-II was also higher in the MG-63/Dox cells as compared with the control cells (Fig. 1C).

Using a TaqMan probe-based qRT-PCR assay, we determined the expression levels of a panel of miRNAs that have been reported to be associated with osteosarcoma cellular autophagy and chemoresistance, particularly miR-1, miR-30a, 
A
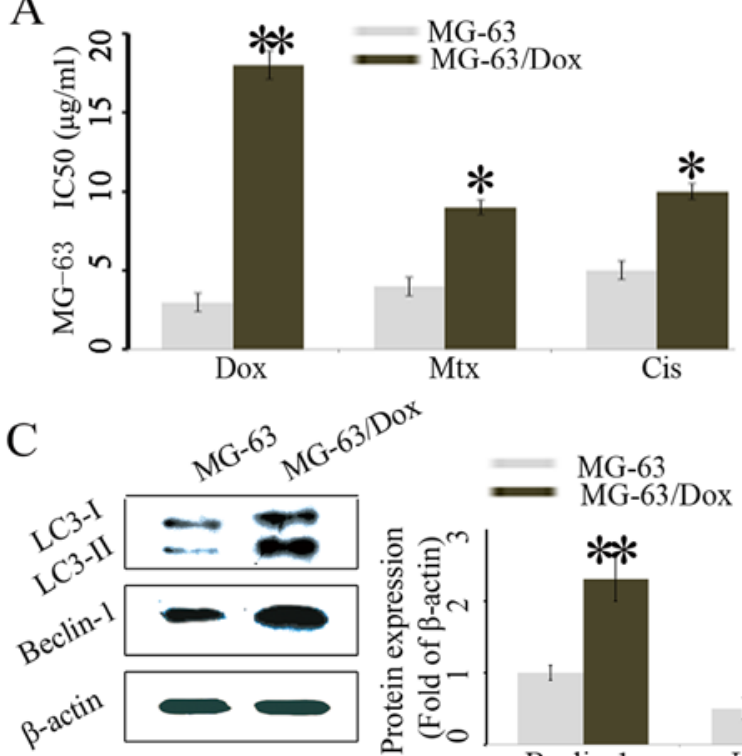

$p^{*}$

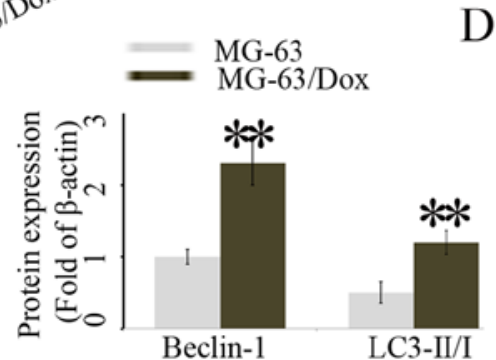

B
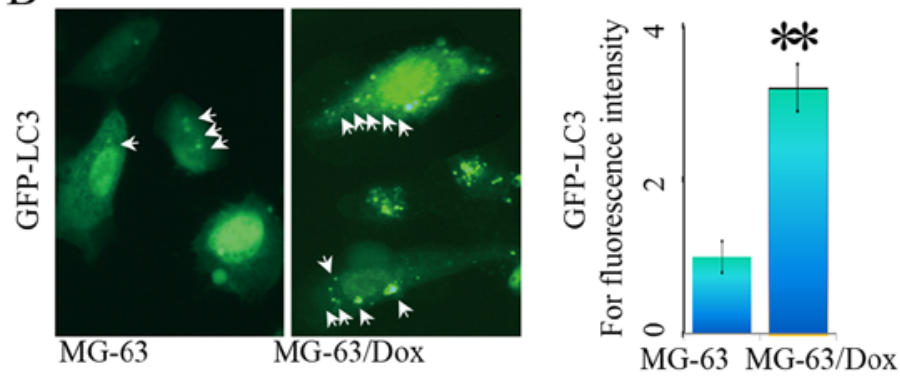

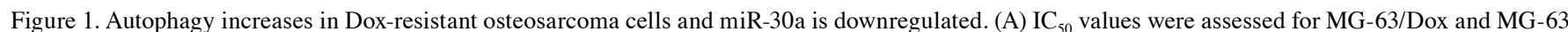
cells after Dox-, Mtx-, Cis-treatment. (B) LC3 puncta were analyzed by a fluorescence microscope in MG-63cells and MG-63/Dox cells. (C) Western blot analysis of LC3-I, LC3-II and Beclin-1 in MG-63 cells and MG-63/Dox cells. Note that after normalization against $\beta$-actin, both Beclin-1 and the ratio of LC3-II/LC3-I significantly increased in MG-63/Dox cells. (D) Expression of miR-1, miR-30a, miR-133a, miR-133b, miR-208b and miR-378c in MG-63 cells and MG-63/Dox cells. The data are presented as the mean \pm SE for three independent experiments. ${ }^{*} \mathrm{p}<0.05 ;{ }^{* *} \mathrm{p}<0.01 \mathrm{MG}-63 / \mathrm{Dox}$ group vs. MG-63 group.

miR-133a, miR-133b, miR-208b and miR-378c (21). As shown in Fig. 1D, among the 6 miRNAs tested, a largest reduction of miR-30a was observed in MG-63/Dox cells compared with the control cells.

miR-30a overexpression suppresses Beclin-1 expression and autophagy is induced by chemotherapy. Subsequently, we assayed the effects of the anticancer agents on the expression of miR-30a. As shown in Fig. 2A and B, anticancer agents significantly suppressed the expression of miR-30a in both MG-63 and MG-63/Dox cell lines. These findings show that miR-30a expression was downregulated during chemotherapy in osteosarcoma cells.

To further investigate the possible role of miR-30a in the MG-63/Dox osteosarcoma cells, we applied lentivirus system to make stable cell lines to overexpress miR-30a on the base of osteosarcoma cell line MG-63/Dox including a blank group (untransfected cells), a Lenti-NC group (cells transfected with the control lentivirus), and a Lenti-miR-30a group (overexpressing of miR-30a). The miR-30a expression levels in these groups were evaluated using qRT-PCR. As expected, the expression of miR-30a was increased in Lenti-miR-30a group cells $(\mathrm{P}<0.05)$ (Fig. 2C). Next, we investigated the effect of miR-30a on autophagy. Results showed that miR-30a overexpression significantly decreased Beclin-1, LC3-I and LC3-II expression, while miR-30a increased p62 protein level (Fig. 2D). A significantly decreased accumulation of LC3-GFP punctae were also confirmed by confocal assay in MG-63/miR-30a cells. These results revealed that overexpressed miR-30a inhibited autophagy in MG-63/Dox cells (Fig. 2E).

To further explore the function of miR-30a in the chemoresistance of osteosarcoma, cell proliferation assays were performed to determine cell growth curve and 50\% inhibition of growth $\left(\mathrm{IC}_{50}\right)$ values following chemotherapy treatment. Overexpression of miR-140-5p did not alter cell growth (Fig. 2F). Remarkably, overexpression of miR-30a dramatically reduced the $\mathrm{IC}_{50}$ values for the three chemotherapeutic agents in MG-63 cells (Fig. 2G).

miR-30a promotes chemotherapy-induced osteosarcoma cell apoptosis via repressing Beclin-1-mediated osteosarcoma autophagy. As shown in Fig. 3A and B, after chemotherapy treatments for $12 \mathrm{~h}$, the ratio of LC3-II versus LC3-I was increased from 2.2-3.5 in the lenti-NC cells in relative to 1.3-1.5 range in lenti-miR-30a group (Fig. 1A). Consistently, the level of Beclin-1 proteins was greatly increased about 1.5-, 1.8- and 2.7-fold in lenti-NC group as compared with 1.1-, 1.23- and 1.28- fold in lenti-miR-30a group, respectively. The above data suggested that miR-30a overexpression led to a downregulation of autophagy responding to chemotherapy in MG-63/Dox osteosarcoma cells. Importantly, apoptosis in MG-63/miR-30a cells was assessed after chemotherapy. Chemotherapy treatment for $12 \mathrm{~h}$ showed 2.6-fold, 1.8-fold, 1.9-fold increase in apoptosis incidence in the Lenti-miR-30a group as compared with the counterparts, respectively.

Furthermore, to determine the role of downregulated autophagy in MG-63/miR-30a cells, autophagy was activated by rapamycin, an autophagy promoter. As a result, rapamycin could partly decrease the Dox-induced apoptosis, suggesting that activating autophagy reversed the effect of miR-30a. Additionally, the apoptotic markers were assessed by western blotting. Compared with the lenti-NC group, the level of cleaved caspase-3 was increased in Lenti-miR-30a group following Dox treatment, suggesting that miR-30a promoted Dox-induced apoptosis. Consistently, rapamycin could partly reduce the expression of cleaved caspase- 3 . 

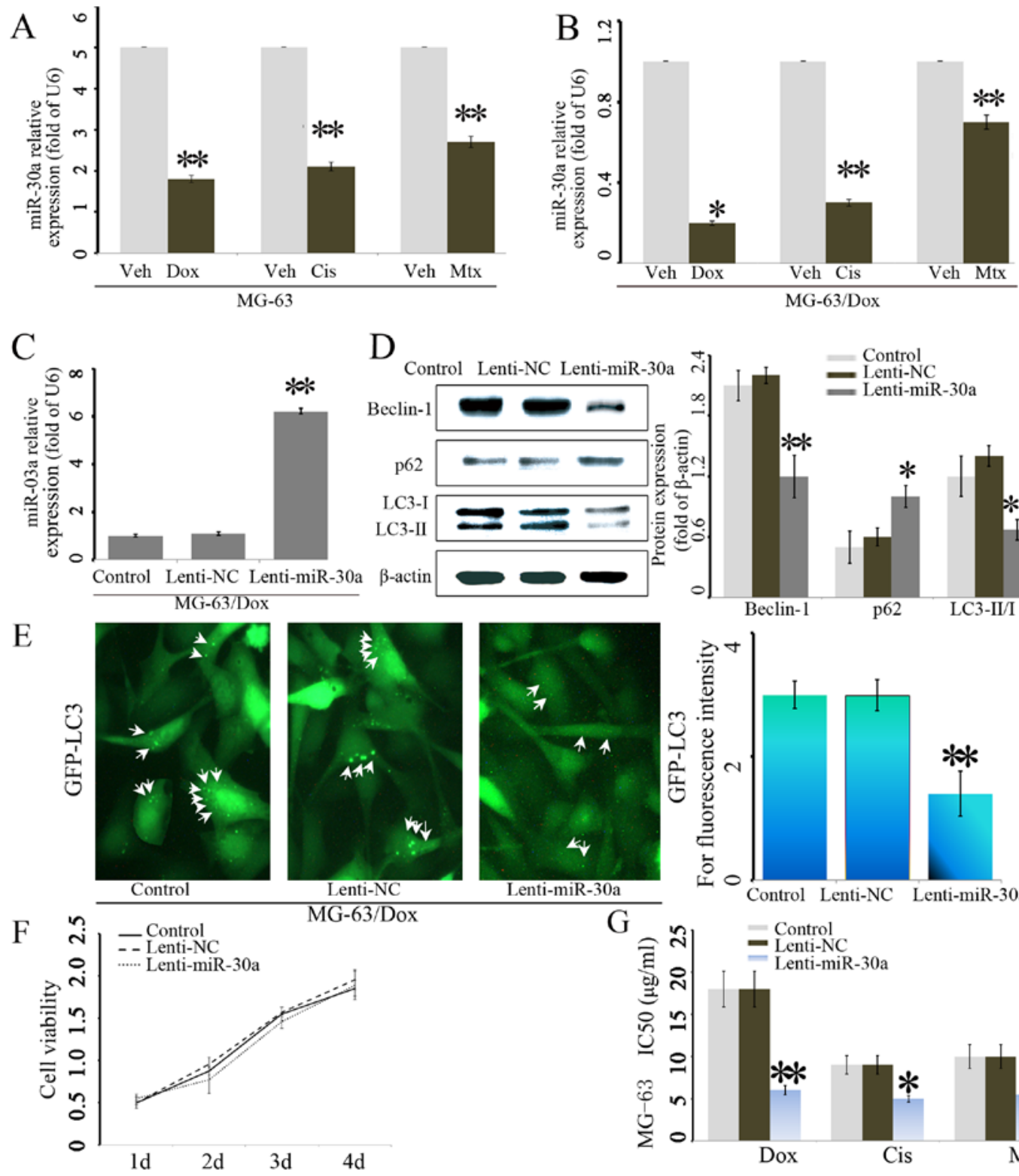

Lenti-miR-30
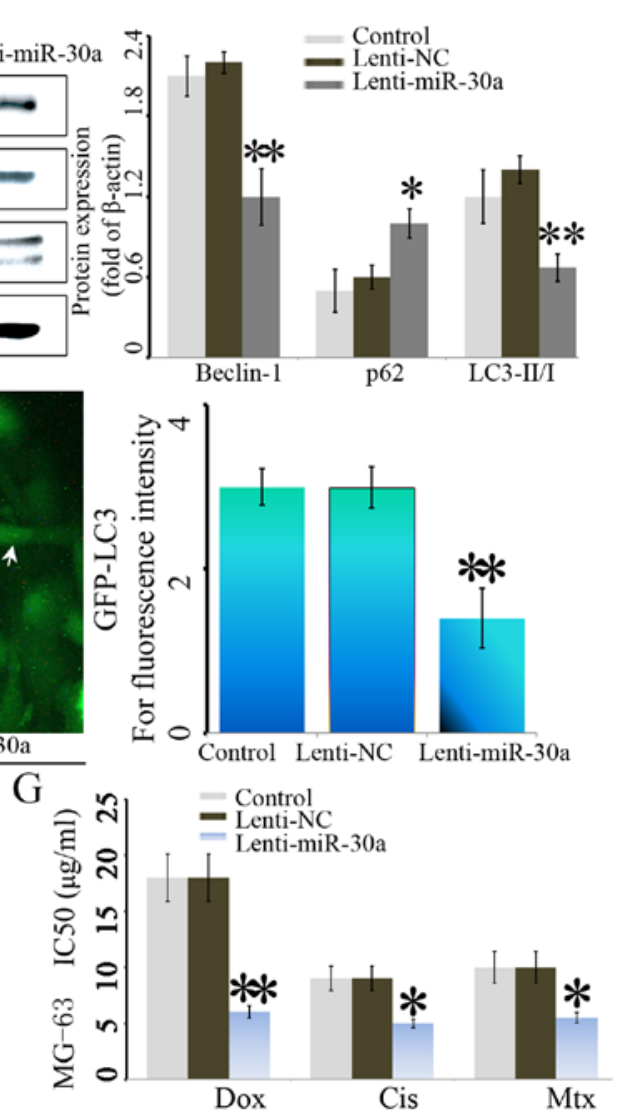

Figure 2. miR-30a overexpression suppresses chemotherapy-induced Beclin-1 expression and autophagy. The expression levels of miR-30a in MG-63 cells (A) and MG-63/Dox (B) after Dox $(0.2 \mathrm{mg} / \mathrm{ml})$, Cis $(20 \mu \mathrm{mol} / \mathrm{l})$, and Mtx $(50 \mathrm{mmol} / \mathrm{l})$ for $12 \mathrm{~h}$. $\mathrm{p}<0.05 ;{ }^{* *} \mathrm{p}<0.01 \mathrm{vs}$. Veh group. (C) miR-30a was overexpressed in MG-63/Dox by lentivirus system and assessed by qRT-PCR after transfection for $24 \mathrm{~h}$. (D) Western blot analysis of LC3-I, LC3-II, p62 and Beclin-1 in cells transfected with or without miR-30a. (E) LC3 puncta were analyzed by a fluorescence microscope in Control, Lenti-NC and Lenti-miR-30a cells. (F) CCK-8 assay for transfected cells. No difference was found in Control, Lenti-NC and Lenti-miR-30a cells. (G) $\mathrm{IC}_{50}$ values were assessed for Control, Lenti-NC and Lenti-miR-30a cells. The data are presented as the mean \pm SE for three independent experiments. ${ }^{*} \mathrm{p}<0.05 ;{ }^{* * *} \mathrm{p}<0.01$ vs. Control group.

These results showed that miR-30a overexpression could promote Dox-induced apoptosis of osteosarcoma cells through suppressing autophagy.

Beclin-1 is a target of miR-30a in osteosarcoma. It has been reported that Beclin-1 is the direct target of miR-30a in the breast cancer cell line MCF-7 (22), and we reasoned that miR-30a may have a similar regulating effect on osteosarcoma. Binding sites for miR-30a in the 3'-UTR of Beclin-1 were identified by bioinformatics analysis (Fig. 4A).

To further assess whether miR-30a was directly targeting Beclin-1 expression through the target site in the 3'-UTR of Beclin-1, reporter constructs containing either the wild-type (WT) Beclin-1 3'-UTR or Beclin-1 3'-UTR with mutation at the predicted miR-30a target sequence were cotransfected into MG-63 cells together with mimic NC, miR-30a mimics, anti-NC or anti-miR-30a. Luciferase reporter assays showed that transduction of miR-30a mimics substantially inhibited the luciferase activity of the wild-type Beclin-1 3'-UTR by $\sim 2 \%$ in MG-63 cells, but had no effect on mutant MG-63 3'-UTR relative to the control (Fig. 4B), and cotransfection with anti-miR-30a significantly increased luciferase activity of WT Beclin-1 3'UTR-compared with anti-NC control (Fig. 4B). Whereas, transfection of the mutant Beclin-1 3'-UTR with anti-miR-30a had no effect on luciferase activity. The above results strongly suggested that Beclin-1 was a direct target of miR-30a in osteosarcoma cells.

To further examine whether miR-30a modulated the expression of Beclin-1 in osteosarcoma, cells were transfected with mimic NC, miR-30a mimics, anti-NC or anti-miR-30a 
A MG-63/Dox (Lenti-NC)
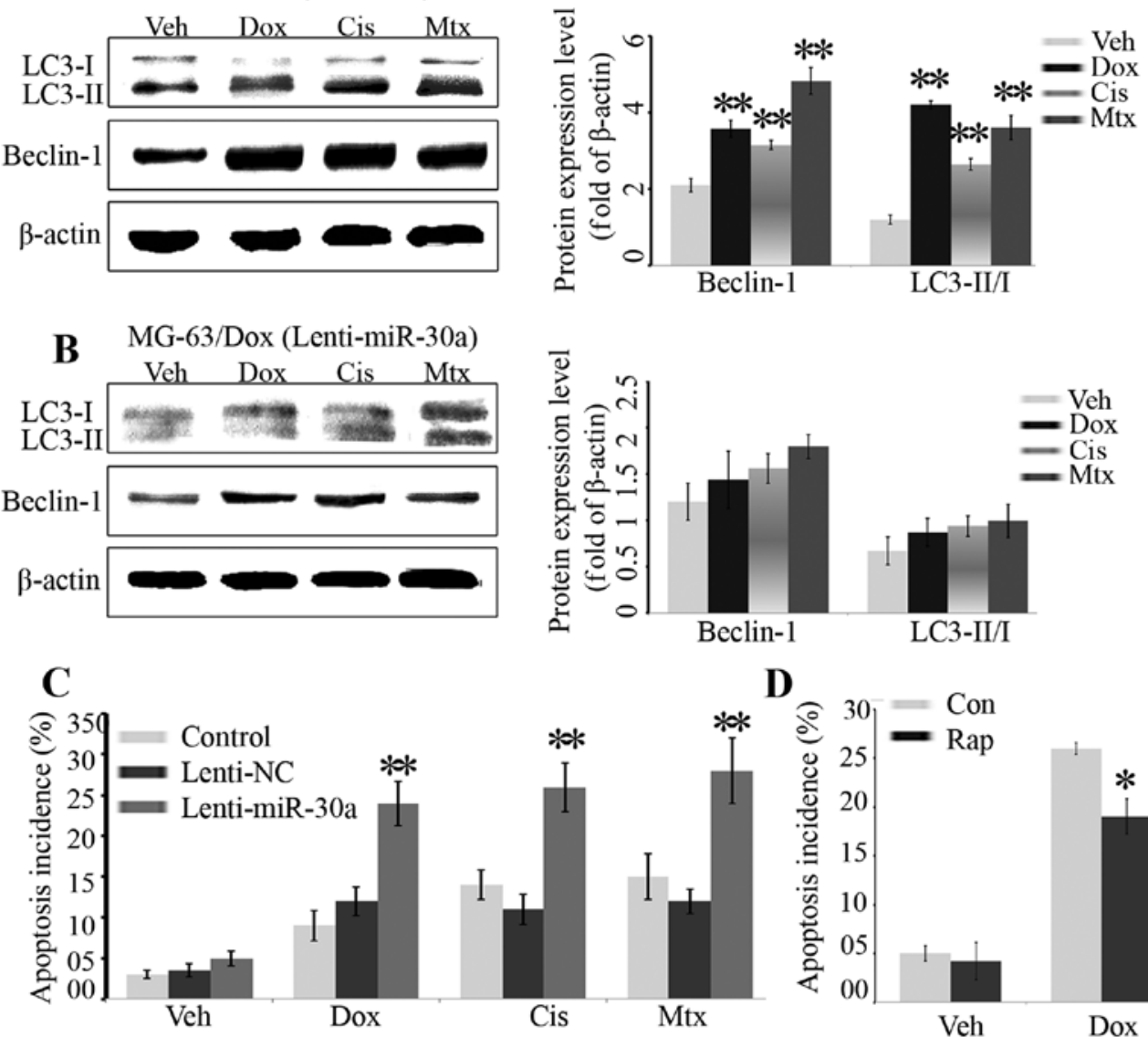

\section{D}
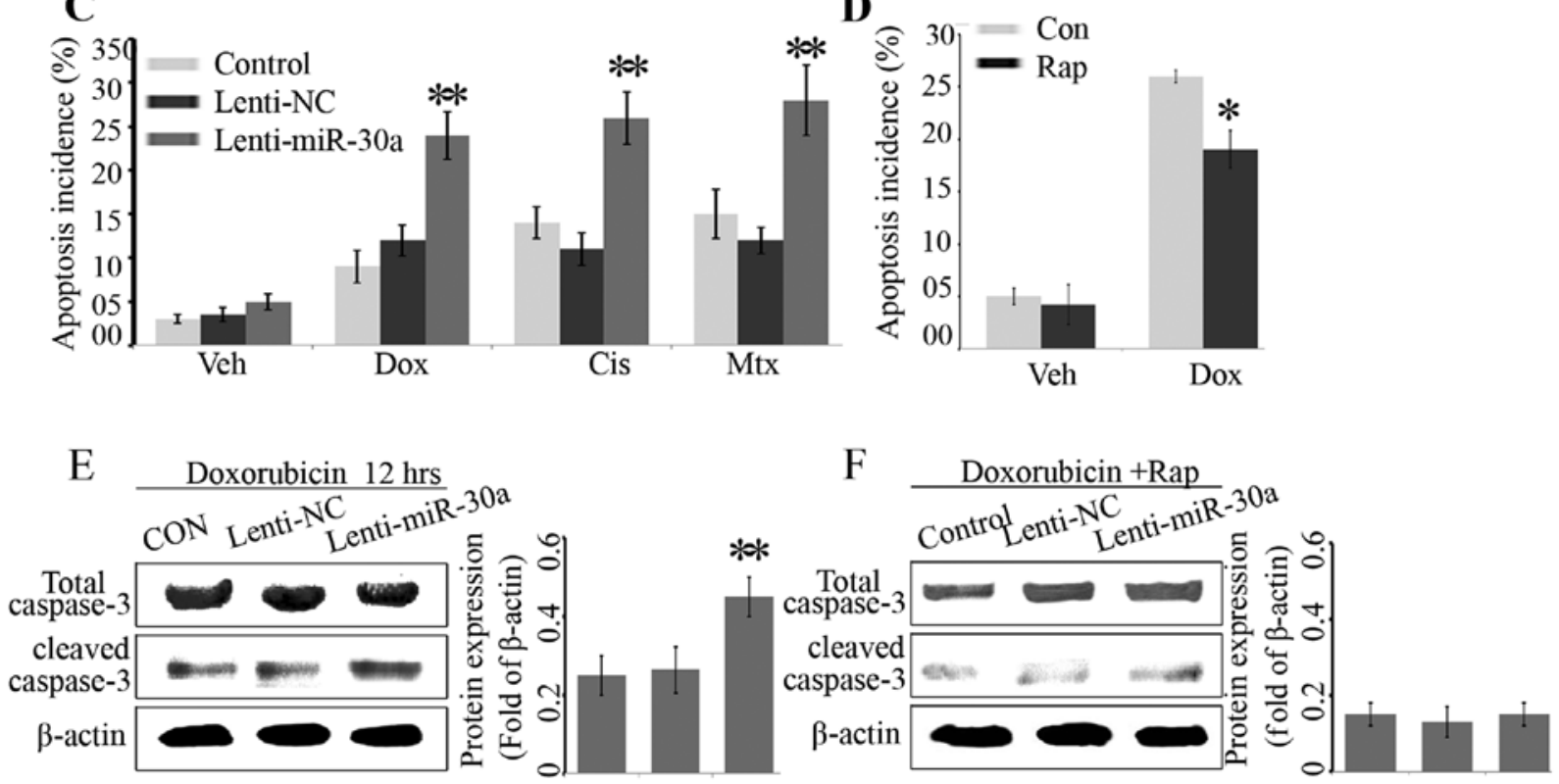

Figure 3. miR-30a promotes chemotherapy-induced osteosarcoma cell apoptosis via repressing Beclin-1-mediated osteosarcoma autophagy. Western blot analysis of LC3-I, LC3-II and Beclin-1. MG63/Dox cells in Lenti-NC group (A) and Lenti- miR-30a group (B) were treated with vehicle (veh, distilled water), Dox $(0.2 \mathrm{mg} / \mathrm{ml})$, Cis $(20 \mu \mathrm{mol} / \mathrm{l})$, and Mtx $(50 \mathrm{mmol} / \mathrm{l})$ for $12 \mathrm{~h} . \mathrm{p}<0.05 ;{ }^{* * *} \mathrm{p}<0.01 \mathrm{vs}$. Veh group. (C) Annexin V-FITC/PI staining for apoptotic cells. MG63/Dox cells in CON, Lenti-NC group and Lenti-miR-30a group were treated with veh (distilled water), Dox ( $0.2 \mathrm{mg} / \mathrm{ml})$, Cis ( $20 \mathrm{mmol} / \mathrm{l})$, and Mtx (50 mmol/1) for $12 \mathrm{~h}$. Subsequently, the apoptosis incidence were quantified by flow cytometry. (D) Annexin V-FITC/PI staining for apoptosis. Cells in Lenti-miR-30a group were pretreated with rapamycin (Rap; $100 \mathrm{nmol} / \mathrm{l}$ ) or without (Con) for $6 \mathrm{~h}$ and then treated with veh (distilled water) or $0.2 \mathrm{mg} / \mathrm{ml} \mathrm{Dox}$ for $12 \mathrm{~h}$. The apoptosis was analyzed by Annexin V-FITC/PI staining using flow cytometry. (E) Western blot analysis of Total caspase-3 and cleaved caspase-3 in Control, Lenti-NC and Lenti-miR-30a groups after $0.2 \mathrm{mg} / \mathrm{ml}$ Dox treatment for $12 \mathrm{~h}$. (F) Western blot for total caspase-3 and cleaved caspase-3. Cells in Control, Lenti-NC and Lenti-miR-30a groups were pretreated with $100 \mathrm{nmol} / 1$ Rap for $6 \mathrm{~h}$ and then treated with $0.2 \mathrm{mg} / \mathrm{ml}$ Dox for $12 \mathrm{~h}$, then analyzed by western blotting. The data are presented as the mean $\pm \mathrm{SE}$ for three independent experiments. ${ }^{*} \mathrm{p}<0.05 ;{ }^{* *} \mathrm{p}<0.01$ vs. Control group.

and then the mRNA expressions of miR-30a and Beclin-1 were analyzed by real-time PCR. Transfection of the miR-30a mimics significantly increased the miR-30a expression and decreased the mRNA level of Beclin-1. Consistently, antimiR-30a had the opposite effects on expression in miR-30a and Beclin-1 (Fig. 4C and D).

\section{Discussion}

With the advancement of neoadjuvent chemotherapy, the global survival rate of osteosarcoma improved to $70 \%$ (23).
However, like most malignant cancers, $\sim 30 \%$ of patients will develop relapse and chemoresistance, which is the major reason for recurrence of osteosarcoma (24). Previously, we investigated the role of Beclin-1 in osteosarcoma, and suggested that the knockdown of Beclin-1 rendered osteosarcoma cells more sensitive to chemotherapy (6). In this study, we observed that chemotherapy induced downregulation of miR-30a in osteosarcoma cells, suggesting that miR-30a might participate in osteosarcoma chemoresistance. Consistently, downregulation of miR-30a has been reported in diverse cancers, and to be involved in tumor cell proliferation and metastasis (11-14). 
A

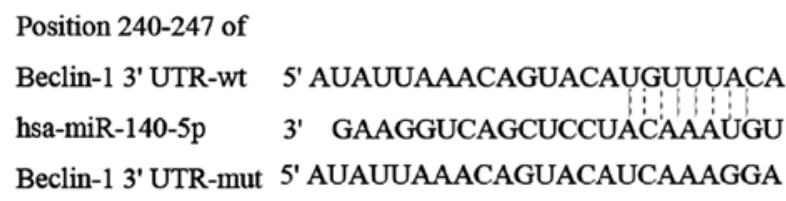

$\mathrm{C}$

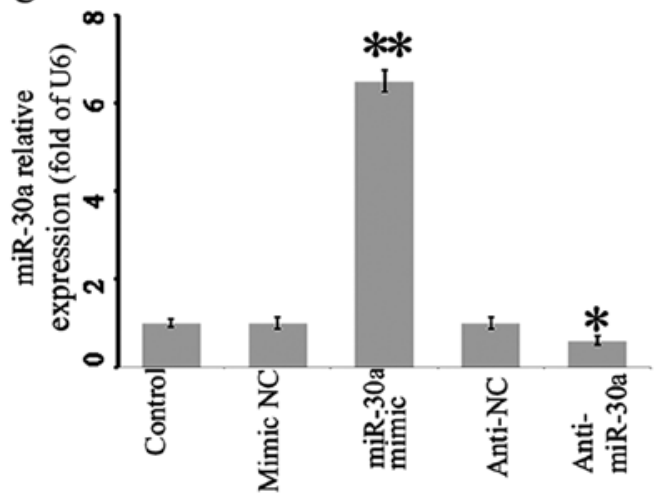

$\mathrm{B}$

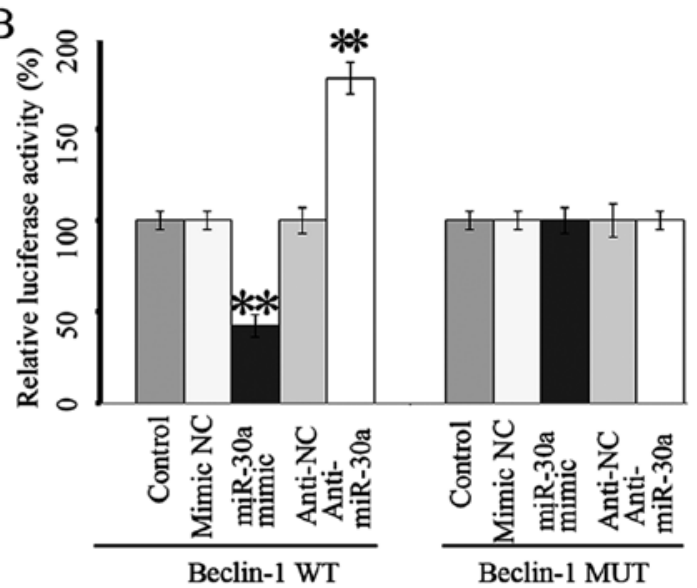

$\mathrm{D}$

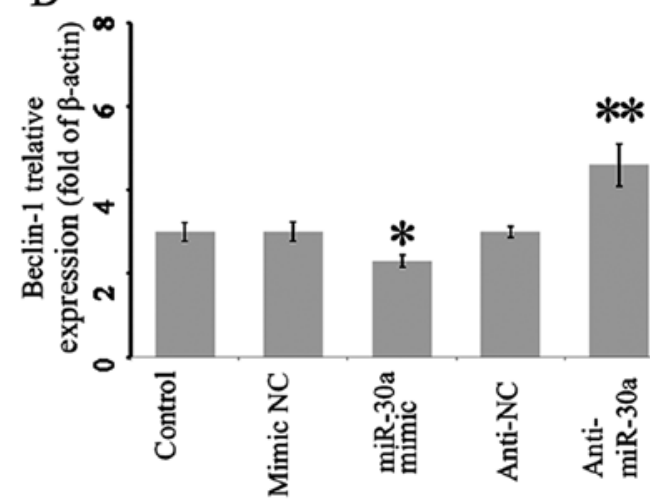

Figure 4. Beclin-1 is a target of miR-30a in osteosarcoma. (A) The predicted binding site of miR-30a on 3'UTR Beclin-1 and the mutated binding site (Beclin-1 3'UTR-mut). (B) A luciferase reporter plasmid carrying Beclin-1 3'UTR-WT or Beclin-1 3'UTR-MUT was transfected into MG-63 cells, co-transfected with mimic NC, miR-30a mimics, anti-NC or anti-miR-30a and luciferase activity was measured. qRT-PCR assay for miR-30a (C) and Beclin-1 (D) expression in MG-63 cells transfected with with mimic NC, miR-30a mimics, anti-NC or anti-miR-30a, respectively. The data are presented as the mean \pm SE for three independent experiments. ${ }^{*} \mathrm{p}<0.05,{ }^{* * *} \mathrm{p}<0.01$, vs. Control.

However, little is known about contribution of miR-30a to chemoresistance of osteosarcoma. Thus, we further investigated the expression of miR-30a in Dox-resistant osteosarcoma. Our results showed there was a significant downregulation of miR-30a and increased autophagy in Dox-resistant osteosarcoma cell line MG-63, which suggested that miR-30a might be a suppressor for autophagy.

Next, to further confirm the biological role of miR-30a in osteosarcoma, miR-30a was overexpressed in Dox-resistance osteosarcoma cell line MG-63/Dox. Real-time PCR confirmed that miR-30a was lowly expressed in MG-63/Dox cells. Overexpression of miR-30a did not alter osteosarcoma cell growth, but it significantly suppressed the autophagy activity of osteosarcoma cells, indicating that miR-30a should be a suppressor for autophagy. Given that autophagy is implicated in chemoresistance (25-27), it was likely that miR-30a downregulation contributed to chemoresistance of osteosarcoma cells by suppressing autophagy, which might explain the parallel downregulation of miR-30a and elevated autophagy in Dox-resistance MG-63 cells.

Autophagy is an essential pathway for cellular homeostasis and recent studies demonstrated that autophagy is implicated in chemoresistance (25-27). However, the regulating mechanism of autophagy in osteosarcoma is still unclear. Our data showed downregulation of miR-30a in Dox-resistance osteosarcoma, consistent with the result in mouse human macrophages (28). Then, it was necessary to clarify whether miR-30a affects the chemoresistance of osteosarcoma. Thus, we next examined the effects of miR-3a on osteosarcoma cell death in the presence of chemotherapy. The data showed that miR-30a strongly reduced the $\mathrm{IC}_{50}$ values and significantly promoted chemotherapy-induced cell death, suggesting that overexpression of miR-30a rendered osteosarcoma cells more sensitive to chemotherapy.

Notably, western blot analysis demonstrated that overexpression of miR-30a enhanced doxorubicin-induced caspase- 3 cleavage, whereas autophagy promoter, rapamycin attenuated doxorubicin-induced caspase- 3 cleavage and reversed the effect of miR-30a. These results strongly suggested that overexpression of miR-30a decreased chemoresistance by suppressing autophagy, leading to increased doxorubicininduced cell death. Several studies have demonstrated that inhibiting autophagy sensitizes tumor cells to chemotherapyinduced cell death, suggesting a key role for the miR-30a/ autophagy loop in chemoresistance of osteosarcoma $(25,29)$. Therefore, it was reasonable that downregulation of miR-30a contributed to chemoresistance of osteosarcoma cells through regulating autophagy. Furthermore, to investigate the mechanism of miR-130a in regulating autophagy, bioinformatics analysis was performed. The results showed that the 3'-UTR 
region of Beclin-1 were the binding sites for miR-30a. Consistently, previous studies demonstrated that Beclin-1 was the directly target of miR-30a (30).

The study of the miR-30a/autophagy loop is based on our previous results and this is the first study of miR-30a in the chemoresistance of osteosarcoma. Our work led to the identification of a novel functional pathway controlled by miR-30a and its direct target, Beclin-1, that regulated chemoresistance in osteosarcoma. Therefore, miR-30a and its target gene pathway may represent new therapeutic opportunities for chemoresistant patients.

\section{Acknowledgements}

This study was supported by the grants from National Natural Science Foundation of Youth Program (no. 81101394), Shanghai Special Fund for Outstanding Young Teachers in Universities (no. JDY10080), Shanghai Renji Hospital Fund for Outstanding Youth (no. RJPY10-010), Shanghai Fund for Young Physician Development (no. 20141051), MedicalEngineering Joint Fund of Shanghai Jiao Tong University (no. YG2014MS51), Shanghai Pujiang Program (no. 15PJD026).

\section{References}

1. Jemal A, Bray F, Center MM, Ferlay J, Ward E and Forman D: Global cancer statistics. CA Cancer J Clin 61: 69-90, 2011.

2. Zhang Y, Duan G and Feng S: MicroRNA-301a modulates doxorubicin resistance in osteosarcoma cells by targeting AMP-activated protein kinase alpha 1 . Biochem Biophys Res Commun 459: 367-373, 2015

3. Xu M, Jin H, Xu CX, Sun B, Mao Z, Bi WZ and Wang Y: miR-382 inhibits tumor growth and enhance chemosensitivity in osteosarcoma. Oncotarget 5: 9472-9483, 2014.

4. Gillet JP and Gottesman MM: Overcoming multidrug resistance in cancer: 35 years after the discovery of ABCB1. Drug Resist Updat 15: 2-4, 2012.

5. He Y, Wang Y, Liu H, Xu X, He S, Tang J, Huang Y, Miao X, Wu Y, Wang Q, et al: Pyruvate kinase isoform M2 (PKM2) participates in multiple myeloma cell proliferation, adhesion and chemoresistance. Leuk Res 39: 1428-1436, 2015.

6. Zhang W, Li Q, Song C and Lao L: Knockdown of autophagyrelated protein 6 , Beclin-1, decreases cell growth, invasion, and metastasis and has a positive effect on chemotherapy-induced cytotoxicity in osteosarcoma cells. Tumour Biol 36: 2531-2539, 2015.

7. Zhang R, Yan S, Wang J, Deng F, Guo Y, Li Y, Fan M, Song Q, Liu $\mathrm{H}$, Weng $\mathrm{Y}$, et al: MiR-30a regulates the proliferation, migration, and invasion of human osteosarcoma by targeting Runx2. Tumour Biol 2015.

8. Jin H and Wang W: MicroRNA-539 suppresses osteosarcoma cell invasion and migration in vitro and targeting Matrix metallopeptidase-8. Int J Clin Exp Pathol 8: 8075-8082, 2015.

9. Mori F, Sacconi A, Canu V, Ganci F, Novello M, Anelli V, Covello R, Ferraresi V, Muti P, Biagini R, et al: miR-181c associates with tumor relapse of high grade osteosarcoma. Oncotarget 6: 13946-13961, 2015.

10. Rodriguez A, Griffiths-Jones S, Ashurst JL and Bradley A: Identification of mammalian microRNA host genes and transcription units. Genome Res 14 (10A): 1902-1910, 2004.

11. Wen XP, Ma HL, Zhao LY, Zhang W and Dang CX: MiR-30a suppresses non-small cell lung cancer progression through AKT signaling pathway by targeting IGF1R. Cell Mol Biol (Noisyle-grand) 61: 78-85, 2015 .
12. Fu J, Xu X, Kang L, Zhou L, Wang S, Lu J, Cheng L, Fan Z, Yuan B, Tian P, et al: miR-30a suppresses breast cancer cell proliferation and migration by targeting Eya2. Biochem Biophys Res Commun 445: 314-319, 2014

13. Zhang Q, Tang Q, Qin D, Yu L, Huang R, Lv G, Zou Z, Jiang XC, Zou C, Liu W, et al: Role of microRNA 30a targeting insulin receptor substrate 2 in colorectal tumorigenesis. Mol Cell Biol 35: 988-1000, 2015.

14. Wang HY, Li YY, Fu S, Wang XP, Huang MY, Zhang X, Shao Q, Deng L, Zeng MS, Zeng YX, et al: MicroRNA-30a promotes invasiveness and metastasis in vitro and in vivo through epithelial-mesenchymal transition and results in poor survival of nasopharyngeal carcinoma patients. Exp Biol Med (Maywood) 239: 891-898, 2014.

15. Yang HZ, Ma Y, Zhou Y, Xu LM, Chen XJ, Ding WB and Zou HB: Autophagy contributes to the enrichment and survival of colorectal cancer stem cells under oxaliplatin treatment. Cancer Lett 361: 128-136, 2015.

16. Wu H, Huang S, Chen Z, Liu W, Zhou X and Zhang D: Hypoxiainduced autophagy contributes to the invasion of salivary adenoid cystic carcinoma through the HIF-1 $\alpha /$ BNIP3 signaling pathway. Mol Med Rep 12: 6467-6474, 2015.

17. Zhou J, Wu S, Chen Y, Zhao J, Zhang K, Wang J and Chen S: microRNA-143 is associated with the survival of ALDH $1^{+} \mathrm{CD}_{133}{ }^{+}$ osteosarcoma cells and the chemoresistance of osteosarcoma. Exp Biol Med (Maywood) 240: 867-875, 2015.

18. Chang Z, Huo L, Li K, Wu Y and Hu Z: Blocked autophagy by miR-101 enhances osteosarcoma cell chemosensitivity in vitro. Sci World J 2014: 794756, 2014.

19. Guo S, Bai R, Liu W, Zhao A, Zhao Z, Wang Y, Wang Y, Zhao W and Wang W: miR-22 inhibits osteosarcoma cell proliferation and migration by targeting HMGB1 and inhibiting HMGB1mediated autophagy. Tumour Biol 35: 7025-7034, 2014.

20. Zhou X, Yuan B, Yuan W, Wang C, Gao R and Wang J: The expression and clinical significance of high mobility group nucleosome binding domain 5 in human osteosarcoma. Tumour Biol 35: 6539-6547, 2014.

21. Zhao H, Li M, Li L, Yang X, Lan G and Zhang Y: MiR-133b is down-regulated in human osteosarcoma and inhibits osteosarcoma cells proliferation, migration and invasion, and promotes apoptosis. PLoS One 8: e83571, 2013.

22. Zou Z, Wu L, Ding H, Wang Y, Zhang Y, Chen X, Chen X, Zhang CY, Zhang Q and Zen K: MicroRNA-30a sensitizes tumor cells to cis-platinum via suppressing beclin 1-mediated autophagy. J Biol Chem 287: 4148-4156, 2012.

23. Luetke A, Meyers PA, Lewis I and Juergens H: Osteosarcoma treatment - where do we stand? A state of the art review. Cancer Treat Rev 40: 523-532, 2014.

24. Huang J, Ni J, Liu K, Yu Y, Xie M, Kang R, Vernon P, Cao L and Tang D: HMGB1 promotes drug resistance in osteosarcoma. Cancer Res 72: 230-238, 2012.

25. Pan B, Feng B, Chen Y, Huang G, Wang R, Chen L and Song H: MiR-200b regulates autophagy associated with chemoresistance in human lung adenocarcinoma. Oncotarget 6: 32805-32820, 2015.

26. Ran X, Yang J, Liu C, Zhou P, Xiao L and Zhang K: MiR-218 inhibits HMGB1-mediated autophagy in endometrial carcinoma cells during chemotherapy. Int J Clin Exp Pathol 8: 6617-6626, 2015.

27. Shteingauz A, Boyango I, Naroditsky I, Hammond E, Gruber M, Doweck I, Ilan N and Vlodavsky I: Heparanase enhances tumor growth and chemoresistance by promoting autophagy. Cancer Res 75: 3946-3957, 2015.

28. Chen Z, Wang T, Liu Z, Zhang G, Wang J, Feng S and Liang J: Inhibition of autophagy by miR-30A induced by mycobacteria tuberculosis as a possible mechanism of immune escape in human macrophages. Jpn J Infect Dis 68: 420-424, 2015.

29. Wang L, Zhang H, Sun M, Yin Z and Qian J: High mobility group box 1-mediated autophagy promotes neuroblastoma cell chemoresistance. Oncol Rep 34: 2969-2976, 2015.

30. Zhu H, Wu H, Liu X, Li B, Chen Y, Ren X, Liu CG and Yang JM: Regulation of autophagy by a beclin 1-targeted microRNA, miR-30a, in cancer cells. Autophagy 5: 816-823, 2009. 